\title{
Advanced care planning in palliative care coordination: the NMC code and record keeping
}

\author{
Bernie St. Aubyn* and Amanda Andrews \\ Senior Lecturer, School of Nursing and Midwifery, Birmingham City University, UK
}

It is essential that nurses adhere to the NMC code in relation to record keeping and documentation in all care arenas. However, this adherence is even more important in the emotional and highly sensitive area of palliative care nursing practise as this group of patients have specific and extra needs that can make their needs more profound and their record keeping requirements more complex. The code explicitly states that nurses must recognise and respond compassionately to the needs of those who are in the last few days and hours of life (NMC 3.2).

This article will consider key elements of palliative care practice in conjunction with the four identified standards of the NMC code (NMC 2015). The four standards are: Prioritise people, practice effectively, preserve safety and promote professionalism and trust. The code clearly stipulates that although the standards may be interpreted differently depending on the practice setting, they are not negotiable or discretionary (NMC 2015). The article will focus on the importance of meticulous record keeping in some of the key areas of palliative care nursing. The areas for consideration are advanced care planning (ACP), advanced decisions to refuse treatment (ADRT) and do not actively resuscitate (DNAR).

\section{Advanced care planning}

About half a million people die each year in the UK [1] and two thirds of these deaths occur in an institutional setting [2]. Despite death being such a common occurrence, however, no one likes talking about death and dying and death can be seen as a failure of the treatment delivered [3]. There is still a lack of professional expertise and reluctance on the part of patients, relatives and professionals to discuss dying even if this could maximise patients' experience of life in the present-day. Advanced care planning enables this difficult subject to be openly discussed between all parties. Perrin and Kazanowski acknowledge that often there are barriers to commencing candid discussions [4]. These barriers might be a reluctance on the part of the patient or family to recognise the seriousness of the presenting condition but could also be due to a lack of knowledge, a surfeit of professionals or the use of incomprehensible medical jargon. These discussions can occur whilst the people involved are still able to effectively and coherently communicate their preferences for future care. There is also an opportunity for informed consent to be obtained and documented for refusal of specific treatments in the event of the patient is not competent to make such a decision in the future $[5,6]$.

Advanced care planning is an aspect of patient care with the potential to enhance quality of life and promote patient autonomy [7]. The treatment and care is often delivered by multidisciplinary teams who can work across local health, social care and voluntary sector services. Communication and sharing of information if therefore crucial to the whole process of establishing and implementing an ACP [7]. The main benefit of ACPs is that they promote timely access to the preferred care voiced by the patient and it can be effectively delivered to meet the patients' expressed needs. The high levels of emotions and distress at this time of life often lead to misunderstandings and conflict between the MDT and the patients and their families. ACP is key in avoiding misconceptions, as the early sensitive discussions about how best to manage the patient will already have been established and documented accurately. The use of an ACP is pivotal in ensuring that professionals recognise and respond compassionately to anxiety and distress in their patients. (NMC 2.6). This also allows for the patient to make their own contribution to their health and wellbeing and encourages partnership working between professional and patient (NMC 2.1, 2.1.2 and 2.4).

The health care professional involved in writing the ACP in conjunction with the patient needs to be mindful that they are complying with the NMC Code (2015). Within the standard Prioritising People, it is necessary to ensure that the interests of the person needing nursing services are put first. This would be achieved by avoiding assumptions about the patients' requirements and making sure that the patients' individual choices were respected (NMC 1.3). The ACP also encourages people to be involved in shared decision making about their treatment and care (NMC 2.3) thus fulfilling the imperative to promote patient autonomy and support patient-centred care [8]. Moreover, the participation of significant others in the ACP process engenders increased knowledge about the preferences of their loved ones care ensuring that they are in a stronger position to participate meaningfully in future critical care decisions [8]. The use of an ACP facilitates the care to be delivered in a timely manner. This resonates with the code which directs that any treatment, assistance or care that the nurse is responsible for is administered without undue delay (NMC 1.4)

Within the standard Practice Effectively there needs to be clear communication between all people involved in the care delivery. Discussions about formulating an ACP need to be conducted using terminology that all relevant people can clearly understand (NMC 7.1). The nurse has a responsibility to meet any specific language or communication needs that people may have (NMC 7.2) and use a range of appropriate verbal and non-verbal methods (NMC 7.3). The adoption of a patient-centred approach will foster a non-discriminatory and culturally sensitive environment (NMC 7.3). Clinicians' behaviour is central to the care process and provides an essential link between

Correspondence to: Bernie St. Aubyn, Senior Lecturer, School of Nursing and Midwifery, Birmingham City University, UK, E-mail: Bernie.St.Aubyn@bcu.ac.uk

Received: December 03, 2017; Accepted: December 22, 2017; Published: December 26, 2017 
interventions and outcomes [9]. It is imperative for all involved to work within a framework of respect and deal with any differences in a professional manner (NMC 9.3)

Once ACP discussions have taken place they are only as useful as the record made of them and they need to be concisely written down or electronically recorded. Moreover, the people consulted in relation to these decisions also need to be noted and identified. Henry and Joseph [5] identify that ACP discussions, with the individual's agreement, should be documented, regularly reviewed and communicated to key persons involved in the care delivery. This resonates with the work done on record keeping by the National End of Life Care Intelligence Network [3] and also with the GMC which goes on to confirm that a record of ACP discussions and decisions should be made available to the patient and shared with others involved in the care to ensure everyone is clear as to what has been agreed (GMC 2010). As part of a regular review the GMC recommend that ACPs should be reviewed and up dated as the patients' situation or views change. The revisions made also need to be shared [3] with the relevant people.

The manner in which the ACPs are recorded needs to adhere to point 10 within the NMC code. The overarching impetus is to keep clear and accurate records relevant to your practice. Within this remit there is a need for the records to be dated and signed and completed contemporaneously. The code stipulates that the records should be accurate and not include any falsification (NMC 10.3) and immediate and appropriate action needs to be taken if someone is aware that these requirements have not been met. This is amplified when considering electronic record keeping by the NELCIN Guidance. Issues can arise and result in complaints against staff where accuracy of records have not been maintained. This guidance resonates with the NMC code as accountability of any erroneous entry lies with the person making the entry (NMC 10.4). If others are aware of inaccuracies they are duty bound to raise their concerns with the responsible clinician as soon as possible [3].

The use of a well formulated and documented ACP can minimise additional medical interventions as patients' conditions alter. This comes under the NMC code remit of preserving safety as these extra interventions may not only conflict with the patients' preferences but could also be of minimal benefit and lead to increased suffering for the individual both physically and psychologically. The ACPs could be seen to give nurses permission to act against their code. It may not be suitable for example for a timely and appropriate referral to be made to others (NMC 13.2) if this does not reflect the preferences agreed in the ACP. Although this action could be considered not to be acting in the patient's best interest there needs to be acknowledgement of the patient's right to refuse treatment (NMC 2.5). All of the interactions involved in the delivery of patient care needs to take place under the mantle of the fourth standard in the code, to Promote professionalism and trust. It would be a given that professionals acted at all times with honesty and integrity, and that they were aware that their behaviour could affect and influence the behaviour of others.

The need for ACPs becomes apparent when an individual develops physical and/or cognitive deterioration and is incapable of accepting or declining medical interventions [10]. An ACP enables an individual to stipulate what their wishes for future medical care they should be rendered incapable of making such decisions.

\section{Advanced decisions to refuse treatment (ADRT)}

An advanced decision has been defined as a specific refusal of treatment(s) in a predefined potential future situation [11]. This term is interchangeable with the terms Advanced Decision, Living Will and Advanced Directive. The legal framework for ADRT is enshrined within the Mental Capacity Act 2005 (NMC 9.2 and 9.3) which came into force in 2007. These advanced decisions represent a chance for people to exert some control over their own destinies to the point where they are no longer able to vocalise their wishes [12]. If the statement is an advanced decision to refuse treatment it is legally binding. This contrasts with any advanced statement to request treatment e.g. placement of care preferences, hygiene regimes etc. which are not legally binding but should obviously be considered. The national end of life strategy (DH 2008) recognises that people in England and Wales have the legal right to refuse specific medical treatment, including treatments that would be classed as life sustaining [7]. ADRT is a complex process requiring legal and ethical considerations that impacts on many areas of nursing practice. Burke v GMC 2005 established the criteria for advanced decision to refuse treatment. These four key point of this criteria are that the patient must be competent and 18 or over, the decisions must be made free from duress or undue influences, the patient must be sufficiently informed and the refusal must apply to the circumstances that subsequently arise [12]. The national end of life strategy's checklist highlights the fact that failure to adhere to the ADRT could potentially result in civil, criminal and/ or professional prosecution [12]. Drafting these ADRTs must not be taken lightly if health care professionals and courts are to take them seriously. It is therefore essential that all documentation compiled relating to ADRTs is clear, concise and accurate. There must be no room for ambiguity as loops holes will allow for them to be ignored readily. The principles of record keeping stipulate that accuracy and clarity are key aspects in nursing documentation to protect both the nurse and the patient [13]. However there is no requirement in law that ADRTs are recorded on approved forms which is an anomaly, so it is even more imperative that the principles of record keeping are adhered to and the code of conduct observed to ensure safety and best practice. In an attempt to give some guidance some organisations produce ADRT templates e.g. the MNDA and Compassion in Dying Organisation. In addition reviews dates need to be set and complied with to ensure the records are contemporaneous in nature and reflective of the current needs of the patient. This is paramount within all aspects of nursing but has particular credence in palliative care as time is a key factor within the patient's end of life care.

\section{DNAR}

As part of the scope 'to refuse treatment' there needs to be some consideration given to the aspect of refusing cardio pulmonary resuscitation (CPR). In instances of a patient dying performing CPR could be an expected part of the dying process. If this would be unlikely to succeed e.g. in a patient with an advanced disease, a management plan is recommended as part of the ADRT and this plan is called a Do Not Attempt CPR (DNACPR) order or Do Not Attempt Resuscitation (DNAR) or Allow Natural Decisions (AND). The benefits, burdens and risks of treatments following successful CPR need also to be considered as such treatment is unlikely to be clinically appropriate. A clear well documented resuscitation order is essential especially if a patient is admitted to hospital acutely unwell to avoid the patient dying in an undignified and traumatic manner.

\section{Conclusion}

Nurses are now operating in a digital age of electronic records and storing of information. Riley discusses the use of Electronic palliative Care Coordination Systems (EPaCCs) to share and record health 
record and care wishes on a digitalised platform [14]. This facilitates the sharing of information accurately across urgent care services e.g. ambulance staff, GP surgeries and out of hours services. Some of these services still rely on hand written documentation which often gets misplaced or left in patients' homes. This can cause confusion when urgent care situations arise and the relevant information is not readily available. EPaCC systems are recommended as a way of improving healthcare documentation and communication within these services. Riley [14] raises a note of caution however relating to digital clinical care planning. If a digital care plan within an EPaCC system is ambiguous, this may result in potential medical errors or delays in treatment. The professionals reading the plan (e.g. the staff in A \& E) would need to employ their own clinical judgement to interpret the recommendation which may differ for the original intention. All records need to provide a place where the professional can add 'free text' as they deliver their care. Nothing can replace the intuition of experienced staff and research has shown that nurses often detect and document subtle changes in their patients before physiological trends become apparent [9].

Finally there is a need for all HCPs to continue to respect each other's roles and strive to learn from other professionals' expertise. This ensures that professionals advance and enhance their own understanding and practice while continuing to provide high quality palliative and end of life care. [4].

This article has looked at some of the elements of palliative care practice within the framework of the four identified standards of the NMC code (NMC 2015). The article has attempted to highlight the importance of meticulous record keeping in some of the key areas of palliative care nursing. Scrupulous record keeping still provides the vehicle to ensure consistence and compassionate care is delivered through clear, concise and unambiguous communication [15-17].

\section{References}

1. Parliamentary and Health Service Ombudsman (2015) Investigations by the Parliamentary and Health Service Ombudsman into complaints about end of life care.
2. Evans CJ, Ho Y, Daveson BA, Hall S, Higginson IJ, et al. (2014) Place and cause of death in centenarians: a population-based observational study in England, 2001 to 2010. PLoS Med 11: e1001653. [Crossref]

3. Public Health England (2015) National End of Life Care Intelligence Network. Palliative care co-ordination: core content. Record Keeping Guidance. NHS, England

4. Perrin KO, Kazanowski M (2015) Overcoming Barriers to Palliative Care Consultation. Crit Care Nurse 35: 44-52. [Crossref]

5. Thomas K, Lobo B, Dettering K (2017) Advance Care planning in End of Life Care. Oxford University Press, Oxford.

6. Waird A, Crisp E (2016) The role of advance care planning in end of life care for residents of age care facilities. Australian Journal of Advanced Nursing 33: 26-34

7. Dempsey D (2014) refusing Treatment: practical, legal and ethical issues. Nursing and Residential Care 17: 454-458.

8. Galambos C, Starr J, Rantz J, Petroski, GF (2016) Analysis of Advance Directive Documentation to Support Palliative Care Activities in Nursing Homes. Health \& Social Work 41: 228-234.

9. Collins SA, Cato K, Albers D (2013) Relationships Between Nursing Documentation and Patients' Mortality. American Journal of Critical Care 22: 305-313.

10. Scandrett KG, Joyce B, Emanuel L1 (2014) Intervention thresholds: a conceptual frame for advance care planning choices. BMC Palliat Care 13: 21. [Crossref]

11. Conroy S, Fade P, Fraser A (2009) Advance care planning: concise evidence -based guidelines. Clinical Medicine 9: 70-79.

12. Avery G (2013) Law and Ethics in Nursing and Healthcare. Sage Publishing. London

13. St. Aubyn B, Andrews A (2015) If it's not written down it didn't happen. Journal of Community Nursing 29: 20-22.

14. Riley J (2017) Transforming end-of-life care - a reimagined urgent care workflow. European Journal of Palliative Care 24: 1-6.

15. Department of Health (2008) End of Life Care Strategy. Promoting high quality care for all adults at the end of life.

16. Gent MJ, Fradsham S, Whyte GM, Mayland CR (2015) What influences attitudes towards clinically assisted hydration in the care of dying patients? A review of the literature. BMJ Support Palliat Care 5: 223-231. [Crossref]

17. Myatt R (2015) Nursing and Midwifery Council revalidation. Nurs Stand 30: 52-59. [Crossref]

Copyright: (C2017 Aubyn BS. This is an open-access article distributed under the terms of the Creative Commons Attribution License, which permits unrestricted use, distribution, and reproduction in any medium, provided the original author and source are credited. 